\title{
Prediction of DAS28-ESR remission at 6 month by baseline variables in patients with rheumatoid arthritis treated with etanercept in Japanese population
}

Naoki Iwamoto ${ }^{1}$, Atsushi Kawakami ${ }^{1}$, Keita Fujikawa ${ }^{1}$, Toshiyuki Aramaki ${ }^{1}$, Shin-ya Kawashiri $^{1}$, Mami Tamai ${ }^{1}$, Kazuhiko Arima ${ }^{1}$, Kunihiro Ichinose ${ }^{1}$, Makoto Kamachi ${ }^{1}$, Satoshi Yamasaki ${ }^{1}$, Hideki Nakamura ${ }^{1}$, Munetoshi Nakashima ${ }^{2}$, Akinari Mizokami ${ }^{3}$, Akiko Goto $^{4}$, Takaaki Fukuda ${ }^{4}$, Naoki Matsuoka ${ }^{5}$, Yukitaka Ueki ${ }^{6}$, Toshiaki Tsukada ${ }^{7}$, Kiyoshi Migita ${ }^{8}$, Fumiko Shoumura ${ }^{9}$, Yojiro Kawabe ${ }^{9}$, Kazutaka Shibatomi ${ }^{10}$, Masanobu Mine ${ }^{11}$, Hiroaki Ida ${ }^{1}$, Tomoki Origuchi ${ }^{12}$, Kiyoshi Aoyagi ${ }^{13}$ and Katsumi Eguchi $^{1}$

${ }^{1}$ Unit of Translational Medicine, Department of Immunology and Rheumatology, Graduate School of Biomedical Sciences, Nagasaki University, 1-7-1 Sakamoto, Nagasaki 852-8501, Japan. ${ }^{2}$ The Japanese Red Cross Nagasaki Atomic Bomb Hospital, Nagasaki, Japan. ${ }^{3}$ Nagasaki Citizen Hospital, Nagasaki, Japan. ${ }^{4}$ Kurume University School of Medicine, Kurume, Japan, ${ }^{5}$ Nagasaki Medical Hospital of Rheumatology, Nagasaki, Japan. ${ }^{6}$ Sasebo Chuo Hospital, Sasebo, Japan. ${ }^{7}$ Isahaya General Hospital, Nagasaki, Japan. ${ }^{8} \mathrm{NHO}$ Nagasaki Medical Center, Nagasaki, Japan. ${ }^{9}$ NHO Ureshino Medical Center, Saga, Japan. ${ }^{10}$ Oita Prefectural Hospital, Oita, Japan. ${ }^{11}$ Suga Orthopedic Hospital, Nagasaki, Japan. ${ }^{12}$ Nagasaki University School of Health Sciences, Nagasaki University, 1-7-1 Sakamoto, Nagasaki 852-8520, Japan. ${ }^{13}$ Department of Public Health, Graduate School of Biomedical Sciences, Nagasaki University, 1-7-1 Sakamoto, Nagasaki 852-8501, Japan

Running title : Prediction of remission induction in RA treated by etanercept by baseline variables

Key Words: Etanercept, Rheumatoid arthritis, MTX, non-MTX nonbiologic DMARDs, DAS28-ESR

Grant Support : This study was supported by a grant from The Ministry of Health, Labour and Welfare, Japan 
Address for correspondence and reprint requests:

Prof. Katsumi Eguchi, M.D., Ph. D.,

Unit of Translational Medicine,

Department of Immunology and Rheumatology

Graduate School of Biomedical Sciences,

Nagasaki University, 1-7-1 Sakamoto,

Nagasaki 852-8501 , Japan

Phone: + 81-95-819-7260

Fax: + 81-95-849-7270

E-mail: eguchi@net.nagasaki-u.ac.jp 


\section{Abbreviations}

ACR: American College of Rheumatology

DAS: Disease Activity Score

DMARDs: Disease modifying antirheumatic drugs

MTX: Methotrexate

RA: Rheumatoid arthritis

TNF $\alpha$ : Tumor necrosis factor $\alpha$ 


\begin{abstract}
Objective. We have tried to determine what baseline variables are responsible for remission induction at 6 months in rheumatoid arthritis (RA) patients treated with etanercept of Japanese population in unselected patients.

Methods. One hundred forty-one patients with RA, etanercept being administrated, were registered. Thirty-four patients were started as etanercept monotherapy, 60 patients as cotherapy with methotrexate (MTX) (MTX cotherapy) and 47 patients as cotherapy with other non-MTX nonbiologic disease modifying antirheumatic drugs (DMARDs) (non-MTX cotherapy), respectively. None of the patients were treated with both MTX and non-MTX nonbiologic DMARDs at entry. The outcome was set as the achievement of Disease Activity Score (DAS) 28-ESR remission at 6 months. We have examined the association of gender, DAS at baseline, MTX cotherapy at baseline, non-MTX cotherapy at baseline and prednisolone use at baseline with the achievement of remission at 6 months by logistic regression analysis.
\end{abstract}

Results. All of the subjects were classified as high disease activity $(\mathrm{N}=109)$ or moderate disease activity $(\mathrm{N}=32)$ at entry. One hundred twenty out of 141 patients (85.1\%) continued the treatment of etanercept at 6 month. Continuation rate was statistically high in MTX cotherapy (93.3\%) as compared with etanercept monotherapy (73.5\%), and tended to be higher than non-MTX cotherapy (85.1\%). Logistic regression analysis identified that MTX cotherapy at entry and moderate disease activity at entry are independent variables toward remission induction at 6 month. Accordingly, DAS28-ESR at 6 month was significantly low in MTX cotherapy as compared with etanercept monotherapy or non-MTX cotherapy. To a lesser extent, DAS28-ESR of non-MTX cotherapy at 6 month was lower than etanercept monotherapy.

Conclusions. In this study of unselected patients, the use of MTX and moderate disease activity at entry was associated with a higher likelihood of response toward etanercept. Non-MTX nonbiologic DMARDs may be alternative in patients with RA to being administrated of etanercept who are intolerant to MTX. 


\section{Introduction}

Randomized controlled trials of the anti-tumor necrosis factor $\alpha$ (anti-TNF $\alpha$ ) therapies including etanercept in rheumatoid arthritis (RA) have shown etanercept to be superior to placebo and to methotrexate (MTX) monotherapy in patients with active RA (1, 2). The trial of Etanercept and Methotrexate with Radiografic Patient Outcomes (TEMPO) study compared etanercept with MTX, starting each as new monotherapy or both as combination therapy, and found the combination to be superior to either drug alone (3). However, the majority of patients in clinical practice who will receive etanercept will already received nonbiologic disease modifying antirheumatic drugs (DMARDs) including MTX yet have ongoing disease activity. In addition, the dosage as well as kinds of nonbiologic DMARDs on the "real-world" of RA in Japan are different from the western countries, thus, an evidence of "real-world" effectiveness of etanercept should be established in Japanese patients.

In this study, we have investigated whether baseline variables at treatment initiation are associated with clinical response to the treatment of etanercept, and shown that MTX cothrepay as well as the moderate disease activity are predictive for remission induction at 6 month. 


\section{Patients and Methods}

Patients. Patients were recruited from Unit of Translational Medicine, Department of Immunology and Rheumatology, Graduate School of Biomedical Sciences, Nagasaki University, Sasebo Chuo Hospital, Kurume University School of Medicine, Gotokai Hospital, The Japanese Red Cross Nagasaki Atomic Bomb Hospital, Nagasaki Citizen Hospital, Isahaya General Hospital, NHO Ureshino Medical Center and NHO Nagasaki Medical Center. The written form of the informed consent, which is approved by the above hospitals, was obtained from each patient. The patients who received with both MTX and non-MTX nonbiologic DMARDs at entry were omitted from this observational study. At entry, 152 patients were recruited, however, 11 patients at evaluation were excluded by combination use of MTX and non-MTX nonbiologic DMARDs. Thus, data of 141 RA patients, who received etanercept for 6 month, were collected. All of the patients fulfilled 1987 criteria of the ACR for RA (4). Disease activity was evaluated by Disease Activity Score (DAS) 28-ESR and all of the patients were high $(\mathrm{N}=109)$ or moderate disease activity $(\mathrm{N}=32)$ at entry. Thirty-four patients were entered as etanercept monotherapy, 60 patients as cotherapy with methotrexate (MTX) (MTX cotherapy) and 47 patients as cotherapy with other non-MTX nonbiologic DMARDs (non-MTX cotherapy), respectively. Non-MTX nonbiologic DMARDs included leflunomide $(\mathrm{N}=8)$, tacrolimus $(\mathrm{N}=5)$, salazosulufapyridine $(\mathrm{N}=8)$, mizoribine $(\mathrm{N}=10)$, bucillamine $(\mathrm{N}=11)$, D-penicillamine $(\mathrm{N}=1)$, tiopronin $(\mathrm{N}=10)$, cyclosporine $A(\mathrm{~N}=3)$ and actarit $(\mathrm{N}=2)$, respectively. Although MTX cotherapy group did not receive other nonbiologic DMARDs at entry, some of non-MTX cotherapy patients received 2 (tacrolimus + salazosulufapyridine; 1 patient, leflunomide + mizoribine; 1 patient, salazosulufapyridine + mizoribine; 2 , bucillamine + tiopronin; 4 , mizoribine + actarit; 1 ) or 3 kinds (bucillamine + tiopronin + D-penicillamine; 1 , bucillamine + tiopronin + cyclosporine A; 1 ) of nonbiologic DMARDs.

Statistical analysis. Distribution of baseline variables were examined by Mann-Whitney's U test, Kruskal-Wallis test and Chi-Square test. Logistic regression analysis was performed to investigate a relationship between baseline variables and clinical efficacy at 6 months. Clinical efficacy was set in the present study as the achievement of DAS28-ESR remission at 6 months (less than 2.6). Etanercept continuation rate at 6 month was also examined. DAS28-ESR at 6 months was judged 
by the last observation carried forward (LOCF) approach. Baseline variables were gender, DAS28-ESR at baseline [High disease activity ( $\geqq 5.1$ ) versus moderate disease activity (3.2 $\leqq$ DAS28-CRP $<5.1$ )] , MTX at initiation (MTX cotherapy), cotherapy with non-MTX nonbiologic DMARDs (non-MTX cotherapy) at initiation and prednisolone at initiation. Prednisolone $>5 \mathrm{mg}$ /day at intiation was also examined for logistic regression analysis. P value less than 0.05 is considered to be significant. 


\section{Results}

Baseline variables of 141 patients with RA treated with etanercept for 6 month and drug survival at 6 month

Table 1 summarized the data. Most of the patients were established disease whose mean disease duration was 10.4 years at baseline. None of the patients were received biologic DMARDs (infliximab, adalimumab, tocilizumab) other than etanercept during the 6 month. All of 141 patients expressed high disease activity $(\mathrm{N}=109)$ or moderate disease activity ( $\mathrm{N}=32)$ at baseline (Table 1). Baseline variables, including age, gender, duration of disease, MTX use at initiation and prednisolone use at initiation were not different between high and moderate disease activity (Table 1). At the end of 6 month follow-up period, 120 patients continued etanercept. The continuation rate at 6 month was $93.3 \%$ (56 out of 60 patients) in MTX cotherapy, 85.1\% (40 out of 47 patients) in non-MTX cotherapy and $73.5 \%$ (25 out of 34 patients) in etanercept monotherapy ( $p=0.004$; MTX cotherapy vs etanercept monotherapy, $\mathrm{p}=0.09$; MTX cotherapy vs non-MTX cotherapy). The reasons for discontinuation, judged by each physician, are the followings: In etanercept monotherapy group, one patient discontinued by lack of efficacy, skin eruption, angina pectoris or perforation of sigmoid colon. Six patients discontinued by respiratory tract infection. One patient complicated with 2 adverse events at the discontinuation. In non-MTX cotherapy group, one patient discontinued by lack of efficacy, itching of skin, compression fracture of the spine or sepsis. Three patients discontinued from respiratory tract infection. In MTX cotherapy group, one patient discontinued by elevation of liver enzymes or respiratory tract infection. Two patients discontinued by skin eruption.

MTX cotherapy and moderate disease activity at initiation are independent predictors for DAS28-ESR remission at 6 months

Table 2 shows the data of logistic regression analysis. As shown in Table 2A, MTX cotherapy and moderate disease activity at baseline were independent predictors toward remission induction at 6 month. The similar results were obtained if the dosage of prednisolone at initiation was limited as $>5 \mathrm{mg} /$ day (Table 2B). Other variables of gender, non-MTX cotherapy, prednisolone at initiation did not reach to statistical significance. Accordingly, baseline variables among MTX cotherapy, non-MTX cotherapy and etanercept monotherapy were similar except for the slight difference of 
age at initiation (Table 3). We have examined the change of DAS28-ESR during 6 month. DAS28-ESR at initiation did not differ among the 3 treatment groups, however as expected, DAS28-ESR at 6 month was significantly low in MTX cotherapy as compared with etanercept monotherapy ( $\mathrm{p}<0.0001$ ) and non-MTX cotherapy $(\mathrm{p}=0.009)$ (Figure 1). In addition, DAS28-ESR at 6 month of non-MTX cotherapy was statistically low as compared with etanercept monotherpy $(\mathrm{p}=0.04)$ (Figure 1$)$. Rate of DAS28-ESR remission at 6 months was 26.7\% (16 our of 60) in MTX cotherapy, 12.8\% (6 out of 47) in non-MTX cotherapy and 8.9\% (3 out of 34) in etanercept monotherapy , which was significantly high in MTX cotherapy ( $\mathrm{P}=0.03$, MTX cotherapy vs etanercept monotherapy). 


\section{Discussion}

The results of this study support the benefit of the combined use of etanercept with MTX, which performed better than etanercept alone or etanercept in combination with non-MTX nonbiologic DMARDs. This is a reasonable, but provides an important information in clinical practice in Japan since the MTX dosage is quite low and non-MTX nonbiologic DMARDs is different as compared with western countries. The trend of non-MTX cotherapy is better than etanercept monotherapy is also similar to the observational study of western countries described by Hyrich et al (5) and Kristensen et al (6). All of the RA patients, etanercept being introduced, will not be tolerant to nonbiologic DMARDs, however, physicians are needed to recognize that MTX and, to a lesser extent, non-MTX nonbiologic DMARDs improve the efficacy of etanercept.

Male gender is selected for the predictor of remission induction by TEMPO study whereas is not found in the present study. MTX dosage is clearly different between the two groups and TEMPO study does not include RA patients treated with non-MTX nonbiologic DMARDs, which may explain the different results.

Systemic administration of low dose glucocorticoids are effective in relieving short term signs and symptoms in patients with RA although its role in the disease outcome remains to be obscure (7). This study does not provide an efficacy of low dose glucocorticoids in patients with RA treated by etanercept. Preferably, treatment of the RA with glucocorticoids is temporary because of the risk of side effects and lack of the add-on effect to etanercept.

TEMPO study has identified lower disease activity at initiation is more likely to reach remission. Accordingly, moderate disease activity at entry is predictive toward remission induction as compared with the patients of high disease activity. Similar results are also obtained in Japanese RA patients treated with infliximab (8), suggesting that this demography may be common indicators for good therapeutic response of DMARDs in patients with RA.

There are limitations to comparing treatment outcomes based on observational data, since the decision to treat patients is not random, but is highly dependent on a number of factors, such as disease severity, patient choice and compliance, and comorbidities. Similary, the decision to stop therapy because of inefficacy or adverse events in this "real-world" study was at the direction of the rheumatologist and was not subject to strict protocol. However, we still found a significant difference between the combined 
use of etanercept plus nonbiologic DMARDs, especially MTX as compared with etanercept monotherapy and moderate disease activity at initiation to the achievement of good therapeutic efficacy. Our data provide clinical benefit for etanercept user in clinical practice.

All authors declared non conflict of interest. 


\section{Acknowledgements}

We thank Miss Kuniko Matsushita for excellent practical assistance. 


\section{Figure Legends}

Figure 1. Change of DAS28-ESR in patients with RA treated by MTX cotherapy, non-MTX cotherapy and etanercept monotherapy during 6 month. DAS28-ESR after entry was calculated by LOCF approach. DAS28-ESR at entry were not different among MTX cotherapy, non-MTX cotherapy and etanercept monotherapy. At 6 month, DAS28-ESR was significantly decreased in MTX cotherapy as compared with etanercept monotherapy and non-MTX cotherapy. In addition, DAS28-ESR at 6 month of non-MTX cotherapy was statistically low as compared with etanercept monotherpy. ${ }^{* *} \mathrm{p}<0.0001$ : MTX cotherapy vs etanercept monotherpy * $\mathrm{p}=0.009$ : MTX cotherapy vs non-MTX cotherapy. $\quad \# p=0.04$ : non-MTX cotherapy vs etanercept monotherapy. The differences were examined by Mann-Whitney’s U test. 


\section{References}

1.Moreland LW, Schiff MH, Baumgartner SW, Tindall EA, Fleischmann RM, Bulpitt $\mathrm{KJ}$, et al. Etanercept therapy in rheumatoid arthritis. A randomized, controlled trial. Ann Intern Med. 1999; 130: 478-486.

2.Weinblatt ME, Kremer JM, Bankhurst AD, Bulpitt KJ, Fleischmann RM, Fox RI, et al. A trial of etanercept, a recombinant tumor necrosis factor receptor:Fc fusion protein, in patients with rheumatoid arthritis receiving methotrexate. N Engl J Med. 1999; 340: 253-259.

3.van der Heijde D, Klareskog L, Landewé R, Bruyn GA, Cantagrel A, Durez P, et al. Disease remission and sustained halting of radiographic progression with combination etanercept and methotrexate in patients with rheumatoid arthritis. Arthritis Rheum. 2007; 56: 3928-3939.

4.Arnett FC, Edworthy SM, Bloch DA, McShane DJ, Fries JF, Cooper NS, et al.: The American Rheumatism Association 1987 revised criteria for the classification of rheumatoid arthritis. Arthritis Rheum. 1988; 31: 315-324.

5.Hyrich KL, Watson KD, Silman AJ, Symmons DP; British Society for Rheumatology Biologics Register. Predictors of response to anti-TNF-alpha therapy among patients with rheumatoid arthritis: results from the British Society for Rheumatology Biologics Register. Arthritis Rheum. 2006; 54:1786-1794.

6.Kristensen LE, Kapetanovic MC, Gülfe A, Söderlin M, Saxne T, Geborek P. Predictors of response to anti-TNF therapy according to ACR and EULAR criteria in patients with established RA: results from the South Swedish Arthritis Treatment Group Register. Rheumatology 2008; 47: 495-499.

7.Saag KG, Criswell LA, Sems KM, Nettleman MD, Kolluri S. Low-dose corticosteroids in rheumatoid arthritis. A meta-analysis of their moderate-term effectiveness. Arthritis Rheum. 1996; 39: 1818-1825.

8.Tanaka Y, Takeuchi T, Inoue E, Saito K, Sekiguchi N, Sato E, et al. Retrospective clinical study on the notable efficacy and related factors of infliximab therapy in a rheumatoid arthritis management group in Japan: one-year clinical outcomes (RECONFIRM-2). Mod Rheumatol. 2008; 18: 146-152. 
Table 1. The characteristics of RA patients at entry

\begin{tabular}{|c|c|c|c|c|}
\hline \multicolumn{2}{|c|}{ Baseline characteristics } & \multicolumn{3}{c|}{ Baseline DAS28-ESR } \\
\hline & All patients & High group & Moderate Group & $\mathrm{p}$ value \\
\hline No. of patients & $\mathrm{N}=141$ & $\mathrm{~N}=109$ & $\mathrm{~N}=32$ & - \\
\hline age, y.o., mean \pm S.D. & $56.8 \pm 13.9$ & $56.0 \pm 14.3$ & $59.5 \pm 11.9$ & $\mathrm{p}=0.22$ \\
\hline gender (male/female) & $32 / 109$ & $24 / 85$ & $8 / 24$ & $\mathrm{p}=0.73^{*}$ \\
\hline duration of disease & $10.4 \pm 8.9$ & $9.9 \pm 8.7$ & $12.0 \pm 9.4$ & $\mathrm{p}=0.47$ \\
\hline DAS28-ESR, mean \pm S.D. & $5.85 \pm 1.06$ & $6.28 \pm 0.73$ & $4.39 \pm 0.55$ & $\mathrm{p}<0.01$ \\
\hline High (>5.1) & $\mathrm{N}=109[77.3 \%]$ & $\mathrm{N}=109$ & - & - \\
\hline Moderate (3.2-5.1) & $\mathrm{N}=32[22.7 \%]$ & - & $\mathrm{N}=32$ & - \\
\hline MTX use at baseline & $60 / 81$ & $49 / 60$ & $11 / 21$ & $\mathrm{p}=0.20^{*}$ \\
\hline (Yes/No) & $\mathrm{N}=124[87.9 \%]$ & $\mathrm{N}=99[90.8 \%]$ & $\mathrm{N}=25[78.1 \%]$ & $\mathrm{p}=0.06^{*}$ \\
\hline PSL use at baseline & $\mathrm{N}=72[51.1 \%]$ & $\mathrm{N}=57[52.3 \%]$ & $\mathrm{N}=15[46.9 \%]$ & $\mathrm{p}=0.37^{*}$ \\
\hline PSL>5mg at baseline & & & & - \\
\hline
\end{tabular}

Baseline characteristics, including age, gender, duration of disease at baseline, MTX use at baseline and prednisolone use at baseline were not different between DAS28-ESR high $(\mathrm{N}=109)$ and DAS28-ESR moderate patients $(\mathrm{N}=32)$. The distribution is characterized by Mann-Whitney's U test or $*$ Chi-Square test as described in the text. 
Table 2. Logistic regression analysis to estimate remission induction by etanercept

A. Variables: gender, MTX at initiation, nonbiologic DMARDs other than MTX at initiation, prednisolone at initiation, DAS28-ESR

\begin{tabular}{llclc}
\hline \multicolumn{1}{c}{ Baseline variables } & comparison & odds ratio & \multicolumn{1}{c}{ 95\% C.I. } & p value \\
\hline Gender & male/female & 0.76 & $0.22-2.60$ & 0.66 \\
MTX & yes/no & $\underline{4.65}$ & $1.13-19.09$ & $\underline{0.03}$ \\
DMARDs other than MTX & yes/no & 1.47 & $0.31-7.03$ & 0.63 \\
Prednisolone & yes/no & 0.56 & $0.16-2.01$ & 0.38 \\
Disease activity (DAS28-ESR) & moderate/high & $\underline{4.08}$ & $1.43-11.55$ & $\underline{0.008}$ \\
\hline
\end{tabular}

B. Variables: gender, MTX at initiation, nonbiologic DMARDs other than MTX at initiation, prednisolone $>5 \mathrm{mg} /$ day at initiation, DAS28-ESR

\begin{tabular}{llclc}
\hline \multicolumn{1}{c}{ Baseline variables } & comparison & odds ratio & 95\% C.I. & p value \\
\hline Gender & male/female & 0.70 & $0.20-2.43$ & 0.57 \\
MTX & yes/no & $\underline{4.68}$ & $1.14-19.16$ & $\underline{0.03}$ \\
DMARDs other than MTX & yes/no & 1.47 & $0.31-7.05$ & 0.63 \\
Prednisolone > 5mg/day & yes/no & 0.46 & $0.17-1.24$ & 0.13 \\
Disease activity (DAS28-ESR) & moderate/high & $\underline{4.27}$ & $1.52-12.00$ & $\underline{0.006}$ \\
\hline
\end{tabular}

MTX use at initiation and moderate disease activity at baseline are predictive for remission induction. 
Table 3. Comparison of baseline characteristics among MTX cotherapy, Non-MTX DMARDs cotherapy and etanercept monotherapy groups

\begin{tabular}{|c|c|c|c|c|}
\hline & MTX cotherapy & $\begin{array}{c}\text { Non-MTX } \\
\text { DMARDs cotherapy }\end{array}$ & $\begin{array}{c}\text { Etanercept } \\
\text { monotherapy }\end{array}$ & $\mathrm{p}$ value \\
\hline No. of patients & $\mathrm{N}=60$ & $\mathrm{~N}=47$ & $\mathrm{~N}=34$ & - \\
\hline age, y.o., mean \pm S.D. & $54.4 \pm 13.2$ & $59.5 \pm 12.3$ & $57.1 \pm 16.7$ & $\mathrm{p}=0.046$ \\
\hline gender (male/female) & $12 / 48$ & $9 / 38$ & $11 / 23$ & $\mathrm{p}=0.30$ \\
\hline duration of disease & $9.3 \pm 7.9$ & $11.4 \pm 9.0$ & $10.9 \pm 10.4$ & $\mathrm{p}=0.69$ \\
\hline DAS28-ESR, mean \pm S.D. & $5.97 \pm 1.01$ & $5.87 \pm 1.04$ & $5.58 \pm 1.13$ & $\mathrm{p}=0.45$ \\
\hline High (>5.1) & $\mathrm{N}=49[81.7 \%]$ & $\mathrm{N}=37[78.7 \%]$ & $\mathrm{N}=23[67.6 \%]$ & $\mathrm{p}=0.29$ \\
\hline Moderate (3.2-5.1) & $\mathrm{N}=11[18.3 \%]$ & $\mathrm{N}=10[21.3 \%]$ & $\mathrm{N}=11[32.4 \%]$ & $\mathrm{p}=0.29$ \\
\hline PSL use & $\mathrm{N}=52[86.7 \%]$ & $\mathrm{N}=42[89.4 \%]$ & $\mathrm{N}=30[88.2 \%]$ & $\mathrm{p}=0.91$ \\
\hline PSL>5mg & $\mathrm{N}=30[50.0 \%]$ & $\mathrm{N}=25[53.2 \%]$ & $\mathrm{N}=17[50.0 \%]$ & $\mathrm{p}=0.94$ \\
\hline
\end{tabular}

Baseline characteristics, including age, gender, duration of disease at baseline, DAS28-ESR at baseline, distribution of DAS28-ESR and prednisoline use at intiation were not different among MTX cotherapy, Non-MTX DMARDs cotherapy and etanercept monotherapy groups except for the slight difference of age at entry. The distribution is characterized by Kruskal-Wallis test as described in the text. 
Figure 1.

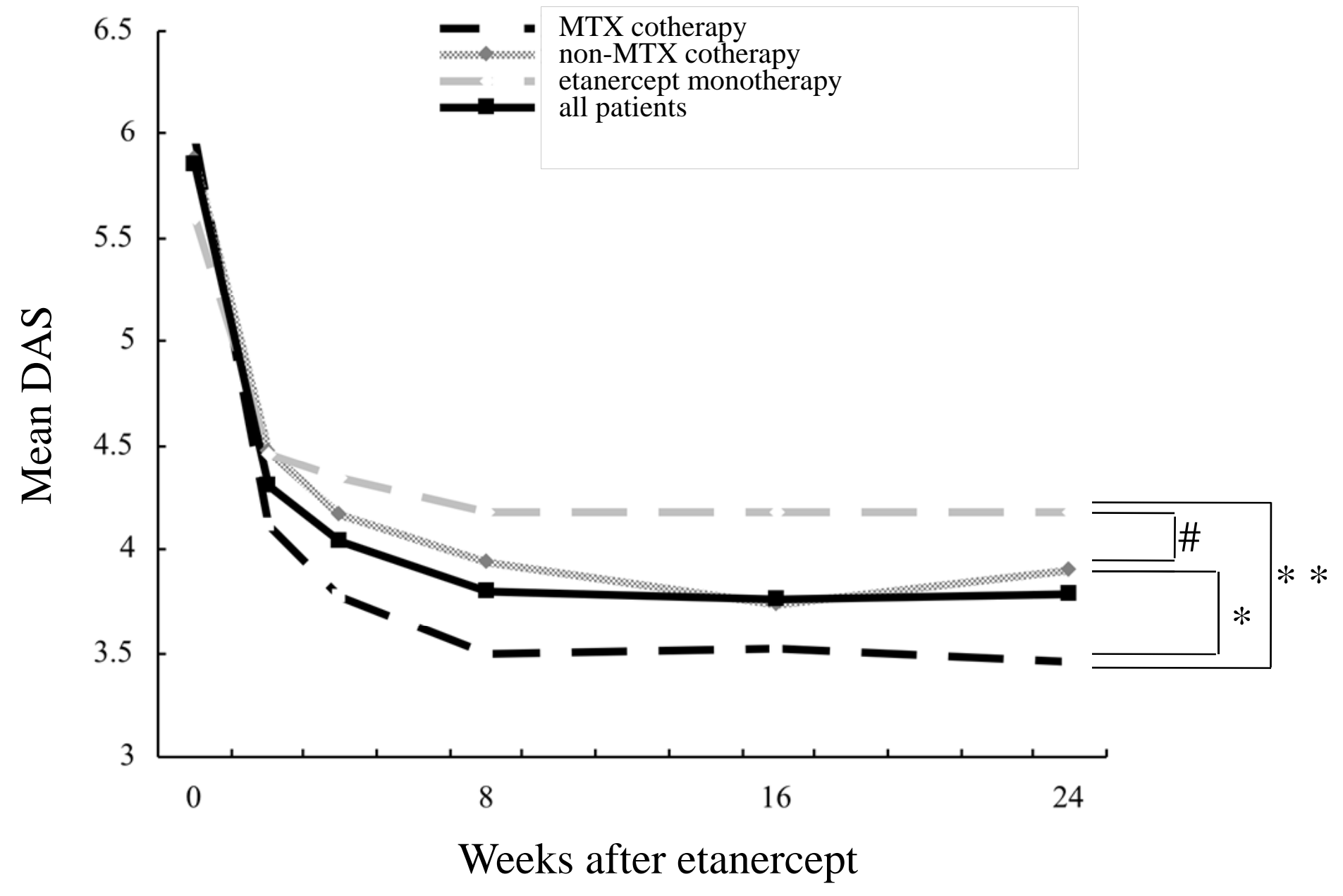

